Kohl : a Journal for Body and Gender Research

Vol. 1, No. 2 (Winter 2015)

\title{
Genre et Éducation Sexuelle Dans le Système Éducatif Marocain
}

Zouhair Gassim

Résumé:

Le système éducatif est un lieu de socialisation par excellence. Outre sa mission de diffusion de savoir, il est un outil de transmission de normes et valeurs qui doivent régir les rapports sociaux, y compris ceux de sexes. Après une analyse genrée du contenu de l'éducation sexuelle dans les programmes scolaires marocains, force est de constater qu'il néglige/oublie l'aspect social et psycho-affectif de la sexualité contenant la dimension de genre. L'absence de ces dimensions qui sont la base de l'identité sexuelle et des représentations, contribue forcément à reproduire les systèmes de classification et de domination déjà inculqués et tatoués dans les mentalités via d'autres espaces de socialisation. 


\section{Contexte:}

Le système éducatif constitue un des hauts lieux de production et de reproduction des rapports sociaux en général, y compris les rapports sociaux de sexes. II n'est pas un simple lieu qui participe à l'instruction ou la diffusion des savoirs, c'est un outil qui transmet les modèles du comportement social.

D'un côté, étant une institution officielle qui vise la formation intégrale de la personne et du futur citoyen, le système éducatif est dans l'obligation de prendre en charge toutes les composantes de la personnalité humaine, y compris la sexualité.

De l'autre côté, la sociologie a tendance à appréhender la sexualité comme, si ce n'est une conséquence, au moins un reflet du genre. BOZON Michel et BUTLER Judith confirmaient I'obligation de l'encadrement social de la sexualité, l'activité des corps dans la sexualité humaine n'est pas une affaire d'instinct mais d'apprentissage social (BOZON, 1999), et les identités sexuées et sexuelles sont construites dans un dialogue avec ce qui est socialement considéré comme possible, souhaitable et compréhensible (BUTLER, 2006).

De ce fait, la sexualité doit faire l'objet d'apprentissages, dans la mesure où elle "contribue à fabriquer le genre: parce qu'elle est à l'origine de l'identification des individu'e.s à un sexe (MIEGEA \& ROUYER, 2012), et un seul, dans un rapport antagonique et complémentaire avec un autre sexe, et un seul autre » (CLAIR, 2012). Sa dimension biologique n'impose pas qu'on la laisse évoluer « naturellement », en dehors du champ de l'éducation et de la connaissance.

Bien que le Maroc ait intégré diverses activités relatives à l'éducation sexuelle dans ses programmes scolaires, leurs analyse est indispensable dans la mesure où l'école reproduit les inégalités sociales à travers des contenus d'enseignement qui privilégient implicitement une forme de culture propre aux classes dominantes, selon la thèse de Pierre BOURDIEU et Jean-Claude PASSERON.

L'intérêt de cette analyse est double. Elle vise, d'une part, à dévoiler les inégalités de genre dans les activités de l'éducation sexuelle véhiculées dans les manuels scolaires. Et d'autre part, plus important, elle vise à ouvrir un autre horizon aux recherches sur l'éducation sexuelle dans les pays arabo-musulmans, qui se sont toutes focalisées sur une seule discipline qui est la Science de Vie et de Terre (SVT), en négligeant/oubliant l'ampleur d'autres disciplines telle l'Éducation Islamique (Él).

Dès lors, et avant d'entamer la description analytique de ces diverses activités (2) nous allons procéder, en premier, à l'identification du corpus, méthode et méthodologie mobilisés (1), pour ensuite dégager les enjeux qui bloquent l'instauration d'une éducation sexuelle égalitaire (3). 


\section{Corpus, méthode et méthodologie}

\section{A. Corpus mobilisé}

Afin de détecter jusqu'à quel point le système éducatif marocain contribue à la promotion du genre via ses programmes scolaires, il est nécessaire de décortiquer les valeurs et les normes véhiculées dans et par les manuels scolaires (b) qui ne sont que la transposition du cadre référentiel officiel (a).

a. Le cadre référentiel englobe l'ensemble des orientations, recommandations, objectifs généraux et spécifiques qui doivent organiser la vie scolaire. Bien que le Maroc se soit engagé dès l'indépendance dans un vaste chantier de réformes du système éducatif, il fallait attendre la fin du siècle dernier pour que le genre soit intégré explicitement dans sa politique éducative. Ainsi, la Charte Nationale d'Éducation et de Formation de 1999 accompagné d'un Livre Blanc, est considérée comme la réforme la plus révolutionnaire dans l'histoire des réformes éducatives au Maroc. C'est la réforme qui a recommandé (dans le Levier 7) la révision des manuels scolaires et leur adaptation au changement sociétal, ce qui a été fait dès 2003.

b. Quant aux manuels scolaires, cette étude s'est focalisée sur deux disciplines scolaires, les Science de la Vie et de la Terre (SVT) et l'Éducation Islamique (Él). Le choix de ces deux disciplines est dû à plusieurs motifs. En se basant sur la multidimensialité de la sexualité, dans la mesure où construire la sexualité est inséparable de la construction d'un savoir scientifique sur la matière, ainsi que la construction de valeurs telles la responsabilité sociale et individuelle (MAMECIER \& MICHARD, 2004).

Pour les SVT, ce sont une discipline qui est très riche d'activités relatives à l'éducation sexuelle dans son aspect biologique et médical comme la reproduction, la puberté, les infections sexuellement transmissibles... Quant à l'Él, souvent marginalisée, elle est la discipline la plus porteuse, implicitement et explicitement, de normes, croyances et valeurs relatives à l'éducation sexuelle, dans la mesure où elle véhicule le référentiel étatique (religieux, politique et socioculturel), tels l'organisation des relations sexuelles, le mariage, la pureté, la chasteté, les droits des femmes, le code civil... L'accouplement de ces deux disciplines, nous semble, pourra dévoiler les différentes dimensions de l'éducation sexuelle au Maroc, à savoir les dimensions biologique, psychosociale, préventive, religieuse et socioculturelle.

Le corpus sélectionné est composé de Quatorze (14) manuels scolaires de différents niveaux répartis comme suit : 
Tableau 1. Répartition des manuels scolaires

\begin{tabular}{|c|c|c|c|c|c|}
\hline \multicolumn{2}{|c|}{ Primaire } & \multicolumn{2}{|c|}{$\begin{array}{c}\text { Secondaire } \\
\text { Collégial }\end{array}$} & \multicolumn{2}{c|}{$\begin{array}{c}\text { Secondaire } \\
\text { Qualifiant }\end{array}$} \\
\hline SVT & ÉI & SVT & Él & SVT & ÉI \\
\hline 2 & 2 & 2 & 3 & 3 & 2 \\
\hline
\end{tabular}

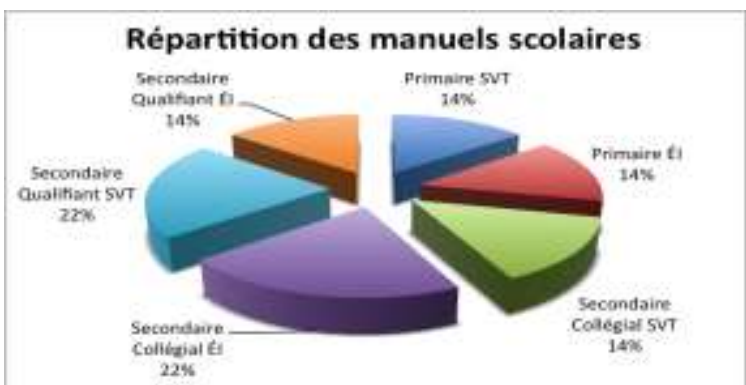

\section{B. Méthode et méthodologie}

L'analyse socioculturelle (genrée) des manuels scolaires et du cadre référentiel est une question complexe. II s'agit de la compréhension des mécanismes d'élaboration des représentations du masculin et du féminin et de dévoiler le système de genre dans ces éléments. Pour ce faire, nous avons choisi trois types d'indicateurs, à savoir rapides, quantitatifs et qualitatifs, répartis en plusieurs grilles d'analyse.

Le premier type d'indicateurs regroupe les éléments d'identification des éléments étudiés. Le second présente un comptage de différentes formes de la représentation du genre dans les activités relatives à l'éducation sexuelle à travers les concepts véhiculés et les types de connaissances transmises. Quant au troisième, il présente une interprétation qualitative des différents éléments énumérés.

\section{Recueil des résultats et analyse}

Après le recueil des différents éléments, nous avons regroupé les constats dans des tableaux récapitulatifs. D'où on a pu enregistrer les résultats sous-cités.

\section{A. Absence totale des auteures femmes}

Dès le départ de cette étude, force est de constater que la discrimination genrée est omniprésente. Parmi 87 auteurs des manuels étudiés, nous avons enregistré une absence totale des femmes.

Tableau 2. Informations générales sur les manuels scolaires

\begin{tabular}{|c|c|c|c|}
\hline & $\begin{array}{c}\text { Proportion du } \\
\text { thème }(\%)\end{array}$ & $\begin{array}{c}\text { Nombre } \\
\text { d'auteurs }\end{array}$ & $\begin{array}{c}\text { La part des } \\
\text { femmes auteures } \\
(\%)\end{array}$ \\
\hline$\underline{\underline{\text { Total }}}$ & 3 & 87 & 0 \\
\hline
\end{tabular}




\section{B. Négligence remarquable de l'éducation sexuelle}

La part des activités relatives à l'éducation sexuelle dans les manuels étudiés ne dépasse plus 3\% (Tableau2). Et ce pourcentage a été calculé sur la base de 14 manuels seulement. Ainsi, la part de ces activités sera plus faible en considérant tous les manuels de l'enseignement scolaire. Ce qui démontre clairement que l'éducation à la sexualité est une discipline marginalisée dans le système éducatif marocain.

\section{Absence de l'aspect social et psycho-affectif}

Le constat enregistré est l'absence totale des concepts qui traduisent l'aspect social et psychoaffectif de la sexualité, comme le montre le tableau suivant :

Tableau 3. Nombres de vocabulaires utilisés.

\begin{tabular}{|c|c|c|c|c|c|c|c|c|c|}
\hline & $\begin{array}{c}\text { Éducation } \\
\text { à la santé }\end{array}$ & $\begin{array}{c}\text { Éducation } \\
\text { sexuelle }\end{array}$ & Sexualité & $\begin{array}{c}\text { Identité } \\
\text { sexuelle }\end{array}$ & $\begin{array}{c}\text { Désir } \\
\text { sexuel } \\
\text { féminin }\end{array}$ & Genre & Orgasme & $\begin{array}{c}\text { Droits } \\
\text { sexuels }\end{array}$ & $\begin{array}{c}\text { égalité } \\
\text { sexuelle }\end{array}$ \\
\hline Total & 34 & 1 & 17 & 0 & 0 & 0 & 0 & 0 & 0 \\
\hline
\end{tabular}

D'une part, les vocabulaires : identité sexuelle, désir sexuel, plaisir, orgasme, qui représentent des caractéristiques psychologiques indispensables de la sexualité, ne trouvent pas place dans les manuels scolaires étudiés. D'autre part, les résultats montrent que l'aspect social est également n'est pas pris en charge. Cet aspect qui n'est que la traduction des valeurs et normes qui doivent régir les relations pendant la vie affective et sexuelle. Ainsi, le genre, les droits sexuels, l'égalité sexuelle, le consentement sont des concepts négligés/omis.

\section{Domination de l'aspect biologique}

Le comptage des types de savoir véhiculé a démontré que la majorité des activités de l'éducation sexuelle cible les dimensions biologique, préventive et instructive.

Tableau 4. Tableau sur les types de savoir véhiculé

\begin{tabular}{|c|c|c|c|c|c|c|}
\hline & Biologique & Préventif/instructif & $\begin{array}{c}\text { Psycho- } \\
\text { affectif }\end{array}$ & Légal/Religieux & $\begin{array}{c}\text { Social } \\
\text { égalitariste }\end{array}$ & $\begin{array}{c}\text { Social } \\
\text { inégalitariste }\end{array}$ \\
\hline $\begin{array}{c}\text { Total } \\
(\%)\end{array}$ & 53 & 31 & 3 & 10 & 0 & 3 \\
\hline
\end{tabular}


La dimension biologique (Anatomie et physiologie des appareils génitaux...), qui représente la majorité, est concentrée dans les manuels des SVT, tandis que les manuels de l'Él contiennent davantage les dimensions préventive et instructive (IST, rapports sexuels hors mariage, chasteté, fidélité, pureté..) à travers les instructions religieuses "faire" et "ne pas faire", en négligeant l'interactivité et condamnant la réflexion.

\section{Enjeux}

Bien que la sexualité soit une composante majeure dans la construction de la personnalité humaine, l'institutionnalisation d'une éducation sexuelle égalitariste est encore tributaire de plusieurs enjeux, politique, religieux, socioculturel et économique.

\section{A. Ambivalence politico-religieuse :}

Le système éducatif n'est pas un simple lieu qui participe à l'instruction ou à la diffusion des savoirs il est également un outil qui transmet les modèles du comportement social : la clé du changement social.

D'un côté, les conservateurs (Islamistes) préfèrent adopter une approche islamique de l'éducation sexuelle. La vision de ce mouvement redouble la loi sociale, en lui conférant un très fort pouvoir d'obligation, de contrainte régulant l'expression du comportement sexuel par les règles, les normes, les interdictions et les tabous qu'elles véhiculent. Ces conservateurs ont justifié le refus d'une telle éducation par: "l'inquiétude que le savoir encourage la promiscuité parmi les adolescents célibataires». ${ }^{1}$ Malek CHEBEL décrivait ces résistances en disant que : «les raffinements de l'activité onirique et sexuelle semblent avoir rencontré une personnalité collective très sensible aux voluptés de la chair» (CHEBEL, 2002).De l'autre côté, les modernistes, y compris les mouvements féministes, rappellent la nécessité d'introduire l'éducation sexuelle comme étant d'une part, une piste parmi autres ciblant la promotion du genre, et d'autre part, une telle éducation va permettre aux jeunes d'acquérir une certaine immunité personnelle, et leur permettre de développer une responsabilité sociale, surtout que le Maroc vit actuellement, selon M. DIALMY, une "transition sexuelle". Cette dichotomie traditionalisme/modernisme, encore objet de polémique, a retardé l'institutionnalisation explicite de l'éducation sexuelle, et a minimisé son contenu au préventif et au biologique.

\section{B. L'éducation sexuelle : affaire privée ou publique?}

\footnotetext{
1 Population Reference Bureau, Jeunesse du monde 1996, Washington.
} 
Bien que le Maroc est conscient de l'aspect public de l'éducation sexuelle, dans la mesure où cette dernière présente un déterminant essentiel dans la prévention des infections sexuellement transmissibles et dans la santé publique en général, on doit pas nier que le "hchouma"(tabou) en reste le caractère dominant, ce qui la situe au cœur d'un conflit d'intérêts: ceux de la personne (intime) et ceux de la société (santé publique et le mœurs).D'une part, dès 1966, l'Etat marocain a essayé de gérer publiquement le contenu de l'éducation sexuelle en l'intégrant dans la planification familiale et dans les programmes de l'éducation en matière de population. Par la suite, elle a été introduite dans les programmes de la santé reproductive et sexuelle, insérée ainsi dans l'éducation sanitaire. Et peu à peu, elle s'est intégrée dans l'éducation formelle sous le nom de l'éducation féminine-familiale du 1985 au 1996, et dans d'autres disciplines telles les sciences naturelles et l'étude islamique jusqu'à maintenant.

En dépit de cette volonté de rendre l'éducation sexuelle une affaire publique, force est de constater que la sexualité au Maroc est encore un espace tabou "hchouma" où le construit social reste le plus pesant (KADRI, BENJELLOUN, KENDILI, KHOUBILA \& MOUSSAOUI, 2012). Le "hchouma" est un sentiment mélangé de pudeur, de honte, de gêne, de culpabilité, d'interdit, de non-dit. "Le mot n'a (même) pas besoin d'être prononcé, la hchouma dicte, contrôle, interdit ; elle se profile derrière bien des actes. (...) C'est un code auquel on se conforme sans réfléchir, et qui légifère (implicitement) toutes les situations de l'existence » (NAAMANE-GUESSOUS, $2001:$ 5).

Dans le même ordre d'idées, "C'est la mère qui est responsable de l'éducation sexuelle des enfants», affirment les sociologues marocains GUESSOUS et DIALMY. D'une part, le père se décharge ainsi de cette tâche difficile, et d'autre part on associe la sexualité à la sphère domestique et privée.

\section{Spécificité et/ou Universalité et/ou Multiculturalité}

Le Maroc, étant un carrefour de différentes cultures, a exprimé maintes fois son inscription à l'universalité, et n'a plus hésité à rappeler son attachement à sa spécificité.D'une part, les réformes adoptées en matière de genre au Maroc sont généralement intervenues dans le cadre du référentiel universel des Droits Humains et plus particulièrement de la Convention sur l'Elimination de toutes les formes de Discriminations à l'Egard de la Femme (CEDEF) et de la Convention des Droits de l'Enfant (CDE). Cette inscription du Maroc dans l'universalité des droits humains a été confirmée, d'une façon éclatante, par l'annonce par SM le Roi de la levée par le Maroc des réserves sur la CEDEF (10 décembre 2008). ${ }^{2}$ En outre, la nouvelle constitution de 2011 a consacré l'attachement du Maroc au référentiel universel et aux conventions universellement reconnues, d'où la confirme volonté à s'inscrire dans l'universalisme.

\footnotetext{
2 Lettre royale adressée au Conseil Consultatif des Droits de l'Homme à l'occasion du 60ème anniversaire de la Déclaration universelle des droits de l'Homme.
} 
D'autre part, le Maroc a exprimé maintes réserves (Chapitre 4 et 7) sur le Programme d'Action de la Convention Internationale sur la Population et le Développement (CIPD) (Le Caire 1994). Ce programme appelle chaque État à incorporer et à observer les droits sexuels et reproductifs dans ses législations nationales. II recommande également aux gouvernements de protéger en particulier « le droit des femmes d'être maîtresses de leur sexualité, y compris leur santé en matière de sexualité et de procréation, sans aucune contrainte, discrimination ou violence, et de prendre librement et de manière responsable des décisions dans ce domaine »(CIPD 1994:6). Ces réserves ont été justifiées par la non-conformité à notre "spécificité" à savoir les normes de la "Charia".

Par ailleurs, Le Maroc est un pays de croisement de cultures. II a connu des influences culturelles amazighes, arabes, juives, méditerranéennes et africaines dont s'enorgueillit la culture marocaine. D'après des études du professeur DIALMY, les représentations et conceptions de masculinité, de féminité et d'éducation sexuelle varient d'une région à une autre (DIALMY, 2009).

\section{La priorité à l'éducation sexuelle ou aux droits socioéconomiques?}

Vu le retard que connait lémancipation des femmes sur le plan socio-économique au Maroc, il n'en reste pas moins que l'éducation sexuelle soit en dernier rang dans les priorités de l'Etat et de la majorité des mouvements féministes.

D'un côté, le dernier rapport du Conseil économique, social et environnemental (CESE) a affirmé que la situation socio-économique des femmes au Maroc est alarmante. ${ }^{3}$

De l'autre côté, ELGAN Elisabeth explique que l'« égalité des sexes, si elle était appliquée à tous les niveaux, changerait radicalement les rapports sociaux non seulement dans la vie privée et dans la vie quotidienne, mais aussi dans le monde du travail, la société civile et la vie politique » (ELGAN, 2012). Dans le même sens, BOURDIEU, HĖRITIER et DELPHY considèrent la sexualité comme source de domination masculine. Cela signifie que les inégalités dans la sphère publique ne sont qu'une prolongation des inégalités initiées dans la sphère privée.

\section{Conclusion:}

${ }^{3}$ Aujourdhui.ma | 30-01-2015, 10:50:00, Par Sara El Majhad. 
Bien que le cadre référentiel officiel du système éducatif marocain se proclame égalitariste, les manuels scolaires demeurent neutres. L'analyse des activités de l'éducation sexuelle montre clairement le degré très élevé de vigilance et de prudence des concepteurs des manuels scolaires. Cette prudence, traduite par le choix de la neutralisation du contenu de cette discipline, a rendu cette dernière très proche de l'information qu'à l'éducation, dans la mesure où elle néglige l'aspect social et psychoaffectif de la sexualité, qui est l'origine des identités sexuées et sexuelles et des représentations. Cette neutralité, selon BOURDIEU, qui disait que rien n'est innocent que le "laisser faire", car laisser faire les normes sans les questionner équivaut à les renforcer, ne peut que contribuer à la reproduction des normes sociales inégalitaires relatives à la sexualité déjà inculquées dans d'autres espaces de socialisation.

Comme l'indiquent Michel BOZON et Henri LERIDON «Paradoxalement, nous manquerions donc de connaissances bien établies sur les comportements sexuels dans nos sociétés. Dans ce domaine, il existe un très gros décalage entre la force des représentations, des idées et des préconstructions issues du monde social et la modestie des connaissances issues des sciences sociales» (BOZON \& LERIDON, 1993). Le Maroc ne fait pas l'exception, d'où l'importance de multiplier et approfondir les recherches, surtout que la transition sexuelle que nous vivons actuellement risquent de coûter chère à la santé publique et à l'Etat. Enfin, il est clair que l'intégration du genre nécessite une approche intégrée et que l'institutionnalisation d'une éducation sexuelle égalitariste serait un outil primordial de déconstruction/reconstruction des rapports sociaux de sexes dans la sphère privée, et qui va servir comme locomotive de l'égalité entre les sexes dans la sphère publique. 
Berger D. \& Jourdan, D. (2008) Education à la santé et prévention des conduites addictive. Quelles formations en IUFM? Toulouse: Presses universitaires du Sud.

BOZON, Michel (1999), Les significations sociales des actes sexuels.

BOZON, Michel \& LERIDON, Henri (1993), Les constructions sociales de la sexualité. in Population, $\mathrm{n}^{\circ} 5$, p. 1180.

BUTLER, Judith, Défaire le genre (Undoing Gender, 2004), traduit de l'anglais Maxime CERVULLE. Paris : Amsterdam, 2006, 311 pages.

CHEBEL, Malek (PRINTEMPS 2002), Sexualité, pouvoir et problématique du sujet en islam. CONFLUENCES Méditerranée - $\mathrm{N}^{\circ} 41$.

CLAIR, Isabelle (2012), Le pédé, la pute et l'ordre hétérosexuel, Agora Débats/Jeunesse, $n^{\circ} 60, p$. 6.

DEVIEILHE, Ėlise (2013), Représentations du genre et des sexualités dans les méthodes d'éducation à la sexualité élaborées en France et en Suède, Thèse de Doctorat en Sociologie, UNIVERSITÉ DE CAEN BASSE-NORMANDIE, FRANCE.

DIALMY, Abdessamad (2009), vers une nouvelle masculinité au Maroc, Conseil pour le développement de la recherche en sciences sociales en Afrique.

ELGAN, Elisabeth (2012), «Regards croisés sur les politiques d'égalité femmes-hommes et leur réception en France et en Suède », in DAUPHIN Sandrine et SENAC Réjane (dir.), Femmeshommes : penser l'égalité. Paris: La Documentation française, p. 196.

Foucault M. Histoire de la sexualité (1976), Tome 1- La volonté de savoir. Paris : Gallimard.

Kadri N, Berrada S, Mchichi Alami K, Manoudi F, Rachidi L, Maftouh A et al. (2009). Mental health of Moroccan women, a sexual perspective.

Kadri N, Mchichi Alami K, Berrada S. La sexualité au Maroc : point de vue de sexologues femmes. Sexologies 2009 ; 19(1): 53-7.

MIEGEA, Y. \& ROUYER, V. (2012), Genre et socialisation de l'enfant: pour une approche plurifactorielle de la construction de l'identité sexuée. En ligne sur www.sciencedirect.com

VĖRONIQUE, Poutrain, L'évolution de l'éducation à la sexualité dans les établissements scolaires, Éducation et socialisation, [En ligne], 36 | 2014, mis en ligne le 15 octobre 2014, consulté le 28 janvier 2015. URL : http://edso.revues.org/951. 